\title{
Appropriate Information Technology In Emerging Economies: An Application For Strategic Decision-
Making In Manufacturing Industries
}

Mehdi Beheshtian Ardakani, (Email: Mehdibeh@ulv.edu), University of La Verne Mohsen Modarres, University of La Verne

Ahmad Ispahani, University of La Verne

\begin{abstract}
Competing in global marketplace has pressured managers respond to shifting market trends by increasing product quality, business process reengineering, and decreasing time to market for new products. Within emerging economies top executives have realized that adoption of appropriate information technologies such a decision support systems (DSS) and group decision support systems (GDSS) have led to changes in the existing organizational structure and communication mechanisms. This paper explores the advantages and constraints of DSS and GDSS in formulating manufacturing strategies in emergent economies. We argued that to fit appropriate information technology to organizational design top executive would benefit from strategic information systems planning process. This process enables top executives to invest in appropriate information system that fits their structural arrangements and organizational culture. Moreover, we explored the impact of DSS and GDSS on executive decision-making capabilities. We also explored the methodology for implementation of appropriate information systems in manufacturing firms in emergent economies.
\end{abstract}

\section{Introduction}

1 nformation Technology is a recognized tool that presents enterprises with the needed flexibility and abilities to meet the new challenges. IT technology is an essential element in the management of firms in emerging economy. It is important to classify IT into possible areas of usage. IT is used to assist in monitoring and controlling organizations, thus improving managerial decision making, increasing organizational flexibility while rising efficiency and effectiveness (Drucker 1989). IT is often used as business communication tools in two different formats. The first is to search for information, and to publish information on the firm which is the organization information systems referred to as MIS. The second is to conduct business decisions making referred to as DSS.

Information technology has also influenced organizational structure by removing the vertical boundaries and flattened the hierarchy. As such, decision making has become decentralized and allowed corporate managers to gather and integrate greater amount of information in strategic decisions. Previous research has focused attention on the capabilities of decision support systems and (DSS) and group decision support system (GDSS) (e.g., Aronson \& Turban, 2001; Carneiro, 2001; Modarres \& Beheshtian, 2003). Past studies, however, have not addressed the fit between appropriate information technology and structural design of the organizations. Moreover, previous researchers have not given attention to appropriate information technologies in industries in emerging economies. In this paper, we explore the advantages of DSS and GDSS in formulating manufacturing strategies in emergent economies. We show that top executive would benefit from strategic information systems planning process. This process enables top executives to invest in appropriate information system that fits their structural arrangements and organizational culture. 


\section{Impact Of Technology On Organizational Structure}

The impact of technology on organizational structure and management role has been the subject of interest to many scholars. The early studies by Thompson (1967), and Blau and Schoenherr (1971) have shown that various types of technologies and computerization of the workplace influence the structural design and management role. More recently, a number of researchers have argued that information technology also tend to have a significant impact on the organizational structure, strategies and communication mechanism (e.g., Bartlett \& Ghoshal, 2000; Yip 2004; Kimberly \& Bouchikhi, 2000). Modarres and Bahrami (1997) argued that investment in information technology would reduce vertical hierarchy by eliminating the middle managers and allow the top executives to access in formation directly and communicate with lower level managers about the strategic decisions. Investment in appropriate information systems has pressured top executives to rethink the organizational arrangements and create coordination and communication mechanisms that lead to competitive advantage in the marketplace.

Karimi, Gupta, and Somers (1996) remarked that contemporary firms are faced with the new challenges. As such, top executives employ information technology in order to gain greater data and information prior to strategy formulation. Recent globalization of manufacturing products has confronted top managers with new competitive challenges such as increasing product quality, decreasing time to market, and business process reengineering. The global competition has also pressured top executives to restructure the organization and reengineer business processes in order to improve organizational effectiveness. In contrast to traditional hierarchical organizational structure and process, managing information technology requires a creative design for cross-functional coordination and sharing of the information for strategic decision making (e.g., Kimberly \& Bouchikhi, 2000).

Information technology transforms the structure of organizations and managements' decision making capabilities. Such transformation requires a careful examination of organizational culture and strategic orientation. Lederer and Sethi (1996) remarked that top executives employ strategic information system planning in identifying appropriate information system for their corporations. Strategic information systems planning has been defined as the process of identifying a portfolio of computer-based applications that will assist an organization in carrying it business plans. The planning for information systems is an important activity for helping information executives and top management identify strategic applications so they can align information technology with structural framework and business needs. In this study, the experiences of 105 planners helped to give a new perspective on how to have more appropriate information systems. The greater the management understanding of culture and structural arrangements of the organizations the higher the success factor in selecting appropriate information systems. Managers in manufacturing industries in emerging economies can employ strategic information systems planning to identify the most desirable information systems applications in which to invest. The characteristics of organizations in emerging economies require effective use or strategic information systems planning. Failure to realize what appropriate technology fits organizational structure and culture may lead to duplicated efforts, incompatible systems, and wasted resources.

Most emerging economies tend to have a rich folk culture, which incorporated traditional themes into its folktales, art, and music. For example in Eastern European economies lack the proper infrastructure and efficient market mechanisms, as such, integration of eastern European management models and information technology with modern Western Europe and industrial world seem improbable without fundamental transformation of the industrial base in Eastern Europe. Similarly, designing effective organizations with interdependent reporting lines require rethinking of the structural arrangements in emerging economies, in particular, in manufacturing industries where the competition can be defined as tight and hypercompetitive. A greater percentage of managers in manufacturing in emergent economies tend to match the application of information technology in their organizations with that of modern industrialized corporations, through engaging in strategic alliances, strategic partnerships and networks. However, without changes in the structural design and culture in their organizations such efforts have failed to produce desired results. Many of these countries also have limited technological capabilities and advancements for improving their production facilities effectively and efficiently. As shown on the Country Industry Analysis, emergent economies are less likely to be able to command stronger financial investment funding in appropriate information technology. 
Decision making about appropriate information technology is known as one of the most important role of executives in manufacturing industries within emergent economies. The existing traditional structure provides redundant written information that is provided by lower level managers to top executives. The traditional written reports are difficult to access or use by managers at all levels for strategic decision making. The structural arrangements in emergent economies reduce the frequency of information use and prolong the length of time associated with the executives decisions making process.

Borthick (1996) described that select of appropriate technology should not be just for the technology sake. The technology has to have a purpose and managers should identify the benefits of such technology prior to investment of resources. Within the emergent economies organizations have been trying to make use of the quickly growing information technologies such as Decision Support Systems (DSS). The more power and complex the technology has become, the more clearly that have been connected to the businesses they support. However, According to Borthick (1996) "no business can escape a fundamental tradeoff: To risk the downside costs and complexity associated with adopting the latest technology, or watch more aggressive competitors profit from its promise (1996:64)."

Farkas (1996) described that in the middle of corporate changes, information services departments are reinventing themselves. The companies are shifting from management evolved during the Industrial Revolution to models more for the information age. However, there are no settled new organizational paradigms, but organizations are refocusing on the core activities of the business. All units are being looked at in a new light and must show how they contribute to the economic performance of the company. For manufacturing organizations in emergent economies selection of appropriate information technology requires changes in management framework and manufacturing strategies. Globalization combined with advanced developments in computing and communication affect all processes relating to the business of the company and the environment.

The strategic decisions in manufacturing industries shift from improving operations of individual units to identifying and improving the processes essential to running the company. As a result operations may be redistributed, eliminated or outsourced. Moreover, top executives should identify appropriate information technology that matches their company's missions, visions and values. The most difficult aspect of selection of appropriate information technology in emergent economies part is helping others to understand the value of the communications and the costs involved in building the infrastructure. To be successful into the future, the organization must standardize network communications select lasting technologies and implementing expandable communicates among many layers of organizational hierarchy.

\section{DSS As Appropriate Information Technology}

Decision Support Systems (DSS) are computer-based systems used as means of extending the capacity of the human mind by allowing convenient access to an enormous amount of data. Theses data could then be entered into some models and used by decision-makers to find best solutions for problems at hand. Although DSS have become widespread in recent years in western countries due to maturity of information technology, in some developing countries decision support system tends to be a luxurious and expensive item. Moreover, organizations in manufacturing industries in emerging economies have faced difficulties in reorganizing their hierarchical structures to fit DSS implementation. This could be attributed to several reasons manufacturing organizations lack of an information system plan or manifesto, expensive software compare to labor cost, and managers' commitment in implementation of DSS which has resulted in lack of support of such efforts. In addition, application of DSS in strategic decision-making has only rarely been seen.

A number of researchers have argued that information technology in general and DSS in particular is impeded by such barriers as lack of "top management support," poor quality IS design, inadequately "motivated and capable" users (Kwon and Zmud, 1987) or restricted access to the system (Poon, P. and Wagner, C., 2001). In the (developing countries), equivalent barriers appear to be often insurmountable (Danowitz, et al., 1995; Knight, 1993; Mahmood, et al., 1995; Nidumolu and Goodman, 1993). While dysfunctional behavior such as little or non use of DSS has been documented in both environments, it seems most troublesome in developing countries in general with little differences in the severity of these problems among these countries (Odedra, et al., 1993). Other researchers go 
so far to say that the diffusion of IT in most developing countries contrast sharply with the experiences in industrialized economies (Jain, R., 1997; Avegrou and Land, 1992; Odedra, et al., 1993; Moussa and Schware, 1992). Hanna, Guy, and Arnold (1995) outline the environmental characteristics of developing countries which influence knowledge management and implementation of information systems.

To remedy these limitations and to enhance quality of top managers' decision making of such system in a major industry, a strategic information system planning need to be developed. The strategic information system planning provides adequate support to the top executive by providing answers to the long-range problems. The ability to answer key questions concerning the long-range problems requires data of many types. The system, then, should be capable of maintaining large quantities of data. It should also have other capabilities such as crossreferencing data and modeling capabilities, and be user friendly.

Although there are a number of implemented and useful DSS, progress toward the strategic use of DSS has been slow (Belardo, D., Duchessi, P. and Coleman, J. R., 1994). Among the reasons for low deployment of these types of systems are the complexity of strategy and the low credibility of computer-based approaches. With regard to the second item, some executives feel that information systems have little to offer them and have been reluctant to accept the systems in their offices (Ledeler, A. L., and Mendelow, A.l. 1988).

\section{Group Decision Support System As Appropriate Information Technology}

The hierarchical structural arrangements in manufacturing industries in emergent economies tend to create barriers to communication. As such, managers tend to make decision at various levels of organizational hierarchy. Group decision support system (GDSS) is designed to reduce the communication barriers. The advantages of top management decision making in a collective fashion regardless of time and geographic place (e.g., Carneiro, 2001). This system will be effective for management models that managers can express their views, and managers have a vote on the selection of the strategic issues to be considered. GDSS has the capability to gather the information from the group members and provide each member with a quasi-decision as the outcome of the managers' meeting. The problem with implementation of GDSS in manufacturing industries in emergent would be the conflict between management styles and organizational culture and requirements for group decision support system (GDSS).

The structural arrangement of the organizations in emergent economies tend to be less appropriate for the consensus gathering, logical analyses of the data available, and voting. Group decision support system provides managers with capabilities such as simultaneous electronic brainstorming (e.g., Briggs, Nunamaker, \& Sprague, 1998), consensus building as results are displayed graphically and statistically, and voting simultaneously (e.g., Aronson \& Turban, 2001). This simultaneous decision process is not effective and does not fit in a hierarchical structure where the information is requested by top management and strategic decisions for resource allocation are made by high level managers.

Group decision support systems have great advantages, however, based on the arguments we made about the appropriateness of DSS and GDSS in the manufacturing industries in emergent economies. DSS characteristics such as the presentation of information and customization of knowledge into a standard report, and retaining information for future decision making and problem solving by top executives make DSS more appropriate information systems for organizations in emergent economies. We will allocate the remaining section of the paper in a methodology that can be considered appropriate for manufacturing industries in emergent economies.

\section{Appropriate Information Technology: New Methodology}

To select appropriate technology we developed four phases:

$\begin{array}{ll}\text { 1. } & \text { Analysis } \\ \text { 2. } & \text { Pilot model building } \\ \text { 3. } & \text { Data base design } \\ \text { 4. } & \text { System implementation }\end{array}$


The analysis process begins with the assignment of a project team headed by a member of executive management who is responsible for the implementation of the system. The executive project team should develop a plan of action that include a description of the approach to be taken, a list of the deliverable documents, and the priorities of the subtasks to be undertaken. It is important from the outset that the method of interaction be specified. This allows managers a chance to influence the design of the system portions that directly affect them. A second phase to design appropriate information technology is to build a series of pilot applications. This phase has multiple objectives. First, it allows testing of the system to see if the user could use it in a real life situation. Second, the pilot applications provide input about the representations type, required from the system. The third phase is critical for management long-range implementation and success of the system. This system allows the top management to conduct cluster analysis and work on the grouping of entities into subjects, useful for the beginning of physical design. The last phase is the implementation of appropriate information technology.

\section{The ROMC Approach}

Any large DSS needs to be considered for its components in terms of ROMC (shim et al, 2002) approach to the system. This system is somewhat unique in that it has a need for shop floor drawing capability, but the requirements for reports for charts and graphs are pretty standard. The discussion by area then is more of a general report than a specific statement of needs for the system.

\subsection{Representations}

The system provides several types of representations. First, there is a report generator capability. In this model the user needs the ability to provide both summary and detail reports on any combination of fields, and in any sequence. The user has the option of specifying report and column headings or allowing the system to generate them from default titles. The reporting function is the same from all applications within the DSS and is accessible at all levels of detail. A second class of representations required is the ability to produce graphs. The standard business graphs including line, bar, scatter plot, and pie charts are considered a minimum. The user is able to exercise control of the format of the graphs by being able to adjust the dimensions, adding of titles and labels, choosing the colors and patterns, and inserting and deleting the data which is to be represented. It is important that the graphs are directly driven from the actual data so that as the background information is changed, the graph will also change without the necessity of inputting new data. While not required for all graphs, the system is capable of outputting presentation quality graphs. Related to the graphics capability, the DSS is able to graphically represent shop floor layouts. This capability is very useful in the determination of capacities and for doing some elementary shop floor planning.

\subsection{Operations}

The Operation of the DSS is primarily the ability to access all of the representations from any part of the system; that is, the graphics capability does not belong to anyone function in the system. It is likely that one area may find more use for a Particular type of representation than does another but all should have access to them. It is also important that the manipulation of representations and models be consistent across all applications. This will allow a user to access a type of data that he may not have used before but to still be comfortable in how it will be represented and in making it meaningful.

\subsection{Memory Aids}

The system provides much help to the user in using the information. One of the basic requirements of the system is an adequate "Help" facility. The user should be able to inquire of the system what all of his options are from any operation that is being attempted. Another facility that should be available to the user is a dictionary of the data items that can be accessed. This dictionary must specify the type of information that is contained in it, the "official" name of the data element, any aliases that may be allowed, and the characteristics of the data. The data Dictionary should allow access by the type of information in addition to the official name and the aliases. A very effective memory aid, which could also be considered a representation, is the capability to represent the structure of 
the Database so that the user has a mental picture of the connections between the relations or record types of the logical database. These representations should show the segments and any network dependencies and the data elements that are contained in each one. Some inquiries may well depend on the structure of the database. In theory perhaps such a facility should not be necessary at least to the end user. But for the data administrator and the new user perhaps such a representation could give a good picture on how the data is organized. It could also provide some insight about why Inquiries might result in unexpected answers.

\subsection{Controls}

The controls of the system are the interfaces that allow the user to "get at" the data and the method of representation that he wants. The dialog serves as a control function in that it controls the interaction between the user and the system. It has already been mentioned that in this system the procedures for creating the various representations are accessible from all parts of the system. This is also true of the models, which are to be used. The user should be able to store these inquiries so that a procedure can be called up without having to enter much information to build the inquiry.

\section{Conclusions}

We discussed the fit between appropriate information system and organizational structure for manufacturing industries in emerging economies. Selection of information technology is a critical task for executive managers and requires redesign of the organizational structure. The focus of the decision-maker, using this system, is on selecting organizational design that fit the information system and enable top executives in making better decisions. We argued that DSS tends to be more appropriate than GDSS in organizations operating in emerging economies. The hierarchical organizational structure can fit in establishment of an information system that automatically extracts the relevant data and make such data available to top managers at various levels for decision making. This requires that the DSS provide an interface tool that facilitates the interaction of model base management system and data base management system, and allows the model designer access database and model base components of the.

Due to similarity of most countries in the emerging economy, this system, when implemented, will allow governments of such countries to adopt similar systems and increase profitability of major industries and eventually help them in privatization of such companies. It also allows implementation of quality assurances and general standards such as ISO 9000 and ISO 14000. Although, a DSS will be successful in emergent economy, a GDDS will face resistance from organizations executive in such economy simply because of nature of the organizational structure, decision making processes, and culture which do not support collaborations and is more hierarchical.

\section{References}

1. Alavi, M., and Joachimsthaler, (1992) "Revisiting DSS implementation research: a meta-analysis of Literature and suggestions for researchers. MIS Quarterly 95-115.

2. Alavi, M., and Napier, H.A., (1984) "An Experiment in Applying the Adaptive Design Approach to DSS Development," Information and Management, 7, pp. 21-28.

3. Bartlett, C. A., \& Ghoshal, S. (2000). Transnational Management. Boston: Irwin McGraw-Hill.

4. Belardo, D., Duchessi, P. and Coleman, J. R., (1994). "A strategic decision support system at Orell Fussli”, Journal of Management Information Systems. Spring 10 (4), 153-150.

5. $\quad$ Bennett, J.L. (1983), Building Decision Support Systems, Addison-Wesley, Reading, Massachusetts

6. Bhatnagar and M. Odedra (ed.) (1992) Social implications of Computer in Developing Countries, Tata McGraw Hill, New Delhi, 26-41.

7. Blau, P. M., and Schoenherr, R. A. (1971) The Structure of Organizations. New York: Basic Books.

8. Borthick, Sandra, (1996) "Strategic Dreams, Real Tactics", Business Communications Review, September.

9. Carlson, J.D., (1979) “An Approach for Designing Decision Support Systems," Data Base, 10,3 and 4, pp. 3-15.

10. Danowitz, A. K., Nassef, Y. \& Goodman, S. E. (1995). "Cyberspace across the Sahara: Computing in North Africa", Communications of the ACM, 38 (12), 23-28. 
11. DeLone, W., and McLean, E.R. (1992). "Information system success: the quest for the dependent variable”, Information Systems Research, 3 (1), 60-95.

12. Farkas-Conn, Irene, (1996) "Linking Information Services to Business Strategies", Bulletin of the American Society for Information Science.

13. Goodman, S.E \& Press, L. I. (1995). "Computing in Vietnam: An Asian Tiger in the Rough", Communications of the ACM 38 (1), 11-16.

14. Hair, Jr., Anderson, R., Tatham, R., and Black, W., (1988). Multivariate data analysis $5^{\text {th }}$ ed._Prentice Hall, NJ.

15. Huber, G.P., and Power, D.J. (1985). "Research notes and communications retrospective reports of strategic level manger: guidelines for increasing their accuracy", Strategic Management Journal, 6, 171-180.

16. Johansen, R., and Swigart, R. (1996). Upsizing the individual in the Downsized Organization: Managing in the Wake of Reengineering, Globalization, and Overwhelming Technological Change, Addison Wesley, Reading, MA.

17. Karimi, Jahangir, Gupta, Yash, and Somers, Toni, (1996) "Impact of Competitive Strategy and Information Technology Maturity on Finn's Strategic Response on Globalization", Journal of Management Information Systems.

18. Kim, K. \& Michelman, J., (1990). "An Examination of Factors for the Strategic Use of Information Systems in Healthcare Industry", MIS Quarterly, June, 201-215.

19. Kimberly \& Bouchikhi, (2000). "Designing the Customized Workplace", in Day, G, Schoemaker, P. J. H (Eds), Managing Emerging Technologies, New York: Wiley, pp: 393-413.

20. Knight, J. (1993). "Contumacious Computer", in W. W. Cotterman \& M. B. Malik (Ed.), Information Technology in Support of Economic Development. Atlanta, GA, USA: Georgia State University Business Press.

21. Krovi, R. (1993). "Identifying the causes of resistance to IS implementation: A change theory perspective", Information \& Management 25, 327-335.

22. Kwon, T. H. \& Zmud, R. w. (1987). "Unifying the Fragmented Models of Information Systems Implementation", in R. J. Boland Jr. \& R. A. Hirscheim (Ed.), Critical Issues in Information Systems Research, (pp. 227-251). New York: John Wiley \& Sons.

23. Ledeler, A.L., and Mendelow, A.L. (1988). "Conceiving top management of the strategic potential of information systems", MIS Quarterly. 12_(4), 525-534.

24. Lederer, Albert, Sethi, Vijay, (1996) "Key Prescriptions for Strategic Information Systems Planning", Journal of Management Information Systems, Summer.

25. Mahmood, M.A., Gemoets, L. A. \& Gosler, M.D. (1995). "Information Technology Transfer and Diffusion to Mexico: A Preliminary Analysis", Journal of Global Information Management, 3 (4).

26. Modarres, M., \& A. Bahrami. (1997). "Corporate downsizing: Preserving the core knowledge base by utilizing expert systems, strategic decision support systems, and intelligent agents", in Knowledge Management and Its Integrative Elements, Liebowitz, J, and L. C. Wilcox (eds). CRC Press: New York.

27. Modarres, M., \& Beheshtian, May 2003. "Enterprise information systems: Integrating Decision Support Systems, Executive Information Systems \& Simulation technology", ISOneWorld Conference, Las Vegas, Nevada.

28. Moormann, J. and Lochte-Holtgreven, (1993) "An approach for an integrated DSS for strategic Planning", Decision Support Systems Vol. 10(4), November, pp. 401-411.

29. Moussa, A. And Schware, R. (1992). "Informatics in Africa: lessons from World Bank Experience", World Development, 20 (12), 1737-1789.

30. Nidumolu, S. R. \& Goodman, S. E. (1993). "Computing in India. An Asian Elephant Learning to Dance", Communications of the ACM, 36 (6), 15-22.

31. Odedra, M., Lawrie, M. Bennett, M. \& Goodman, S. (1993). "Sub-Saharan Africa: A Technological Desert", Communications of the ACM, 36 (2), 22-29.

32. Poon, P. and Wagner, C., (2001). "Critical Success Factors Revisited: Success and Failure Cases of Information Systems for Senior Executives”, Decision Support Systems, 30, 393-418.

33. Rovere, R. L., "Small and Medium-Sized Enterprises and IT Diffusion Policies in Europe: Lessons for Developing Countries", Information Technology in Developing Countries Volume 6, No. 2, April 1996. 
34. Shibanda, G. G. and Musisi-Edebe, I., (2000). "Managing and developing the strategy for Africa' information in global computerization", Library Management 21(5), 228-234.

35. Shim, J., Warkentin, M., Courtney, J., Power, D. J., Sharda, R., and Carldsson, C., (2002) "Past, Present, and future of decision support technology", Decision Support Systems, Vol. 33(2), pp.111-126.

36. Sprague Jr. R. H. and H. J. Watson, (1983) Decision support for management, Upper Saddle River, New Jersey, Prentice Hall.

37. Stevens, J. P., (1992). Applied multivariate statistics for the social sciences $\left(2^{\text {nd }}\right.$ ed.). Hillsdale, NJ.

38. Tebbut, David, (1996) "How New Technology Affects Business", Director, p. 91-103.

39. Thompson, J. D. (1967). Organizations in Action. New York: McGraw-Hill.

40. Weiner, L. R., (1993). Digital Woes: Why We Should Not Be Depending on Software, Addison-Wesley, Reading, MA.

41. Yip, G, S. (2004). Total Global Strategies. New Jersey: Prentice Hall 